\title{
Erratum to: Scheduling jobs on parallel machines to minimize a regular step total cost function
}

\author{
Boris Detienne $\cdot$ Stéphane Dauzère-Pérès · \\ Claude Yugma
}

Published online: 19 August 2011

(C) Springer Science+Business Media, LLC 2011

\section{Erratum to: J Sched \\ DOI 10.1007/s10951-010-0203-Z}

Our paper entitled "Scheduling jobs on parallel machines to minimize a regular step total cost function" appeared online in Journal of Scheduling. It is an extension from the conference article (Detienne et al. 2009). In these works, we missed two studies considering stepwise cost functions in scheduling problems, in which the authors refer to the optimization criterion as stepwise tardiness.

Curry and Peters (2005) investigates a rescheduling problem in a parallel machine environment. The authors examine the tradeoff between schedule stability and tardiness cost, using simulation. A job can be on time or late for 1, 2, 3 or 4 days, thus yielding a stepwise cost function. They also consider machine reassignment costs. Within the simulation, the rescheduling problem is solved using a branch-andprice algorithm. They propose an Integer Linear Program-

The online version of the original article can be found under doi:10.1007/s10951-010-0203-z.

\section{B. Detienne $(\bowtie)$}

Laboratoire d'Informatique d'Avignon, Université d'Avignon et des pays de Vaucluse, 339 chemin des Meinajaries, Agroparc BP 1228, 84911 Avignon Cedex 9, France

e-mail: Boris.Detienne@univ-avignon.fr

S. Dauzère-Pérès · C. Yugma

Department of Manufacturing Sciences and Logistics CMP, École des Mines de Saint-Étienne, Site Georges Charpak, 880 route de Mimet, 13541 Gardanne, France

S. Dauzère-Pérès

e-mail: Stephane.Dauzere-Peres@emse.fr

C. Yugma

e-mail: Claude.Yugma@emse.fr ming (ILP) formulation for a relaxation of the rescheduling problem, in which preemption is allowed. This relaxation focuses on due date assignment, thus it avoids the difficulty of sequencing the jobs in the presence of release dates. Our paper explicitly addresses this issue and proposes an appropriate ILP model.

Sahin (2006) considers the single machine special case of the problem. The author presents industrial applications of the optimization criterion, and two solution algorithms. The first one consists of two ILP formulations: A classical timeindexed formulation, similar to the one we use as a baseline in our paper, and a preemption-based assignment formulation. The second solution algorithm is a LP-based constructive heuristic, improved by a k-exchange local search. In our opinion, a drawback of these methods is that both ILP formulations count a pseudo-polynomial number of variables and constraints, which depends on the length of the scheduling horizon. Consequently, due to memory requirements, the direct resolution of the two ILP models or of their linear relaxations is impossible nowadays for most instances with more than 50 jobs and processing time varying up to 100 time slots, so that the heuristic method cannot be used as well. In our paper, we propose two alternate ILP models whose sizes depend only on the number of jobs and the number of distinct due dates. A drawback of our models and the resulting dedicated solving approach is a degraded performance when the number of due dates increases.

\section{References}

Curry, J., \& Peters, B. (2005). Rescheduling parallel machines with stepwise increasing tardiness. International Journal of Production Research, 43(15), 3231-3246. 
Detienne, B., Dauzère-Pérès, S., \& Yugma, C. (2009). Scheduling inspection operations subject to a fixed production schedule. In Proceedings of the 4th multidisciplinary international conference on scheduling: theory and applications (MISTA 2009) (pp. 581593).
Sahin, G. (2006). New combinatorial approaches for solving railroad planning and scheduling problems. $\mathrm{PhD}$ Thesis, University of Florida, FL, USA. 